\title{
ПУТИ РЕШЕНИЯ ЗАДАЧИ ОЦЕНИВАНИЯ ХАРАКТЕРИСТИК ДВИЖЕНИЯ ОБЩЕСТВЕННОГО ТРАНСПОРТА
}

\author{
А. М. Лукьянов, О. Г. Алдабаева, А. А. Лукьянов \\ Белгородский государственный технологический университет им. В. Г. Шухова
}

Поступила в редакцию 26.04.2018 г.

\begin{abstract}
Аннотация. Для городов характерно наличие большого количества маршрутов движения общественного транспорта. Их количество достигает десятков, а зачастую и сотен. В настоящее время в условиях реальной дорожной обстановки это приводит к несогласованности прибытия транспортных средств разных маршрутов на общие остановочные пункты. В результате чего, зачастую, образуются очереди для въезда и выполнения высадки/посадки пассажиров. Причиной этого является то, что в расписаниях движения определены время выезда с начального/конечного пункта, но отсутствует, как таковой, график движения по маршруту, который практически невозможно просчитать, не зная дорожной реальной обстановки на дорогах. Предлагаемый в работе подход позволяет в автоматическом режиме оценивать многие необходимые для планирования перевозок фактические параметры движения маршрутного транспорта, причём, оценивать их распределение в зависимости от факторов, определяющих интенсивность движения по дорожной сети - сезона, дня недели, времени суток и т. д.

Ключевые слова: дорожное движение, общественный транспорт, навигационные данные, автоматизация контроля движения транспорта, диспетчерская, расписание.

Annotation. For cities is characterized by the presence of many public transport routes. Their number reaches tens, and often hundreds. At present, in conditions of a real road situation, this leads to inconsistency in the arrival of vehicles of different routes to common stop points. As a result, often, queues are formed for the entry and disembarkation / landing of passengers. The reason for this is that the timetables of the movement have determined the time of departure from the start / end point, but there is, as such, no schedule for the route, which is almost impossible to calculate without knowing the real road situation on the roads. The approach proposed in the work allows in an automatic mode to estimate the actual parameters of the movement of route transport necessary for planning the transportation, and to assess their distribution depending on the factors determining the traffic intensity on the road network - the season, the day of the week, the time of day, etc.
\end{abstract}

Keywords: road traffic, public transport, navigation data, traffic control automation, dispatching, schedule.

\section{ПРОБЛЕМНЫЕ ВОПРОСЫ ОРГАНИЗАЦИИ ПАССАЖИРСКИХ ПЕРЕВОЗОК ГОРОДСКИМ АВТОМОБИЛЬНЫМ ТРАНСПОРТОМ}

Для обеспечения эффективного решения задачи организации движения маршрутного транспорта необходимо знать текущую обстановку на дорогах и с высокой степенью точности прогнозировать характеристики транспортных потоков на различных участ2018

() Лукьянов А. М., Алдабаева О. Г., Лукьянов А. А., ках дорожной сети, учитывая при этом время суток, сезонные факторы и другие условия. Данные об условиях движения, предоставляемые в баллах сервисом «Яндекс.Пробки» [1] недостаточны для эффективного планирования движения именно общественного транспорта - они не учитывают движение по выделенным полосам, время высадки/посадки пассажиров и другие факторы. Тем более, что этот сервис внедрён только в некоторых крупных городах.

В таких городах как Великий Новгород, Киров и некоторых других расписания движения общественного транспорта существу- 
ют и доступны для всеобщего обозрения $[2,3]$. Однако они не учитывают особенности движения транспорта в выходные и праздничные дни. Кроме того, даже беглого взгляда достаточно для того, чтобы увидеть, что расписание не учитывает изменения средней скорости движения в течение дня. Что не даёт реальных оснований для использования расписаний для планирования пассажирами своих поездок.

Также следует отметить такие города как Санкт-Петербург, где расписание движение не представлено, а есть только список остановок [4].

Основной причиной фактического отсутствия реальных расписаний движения является недостаток информации об условиях движения городского транспорта.

Перечисленные проблемы характерны для г. Белгорода, в котором насчитывается 93 маршрута (28 - автобусных, 53 - маршрутных такси и 12 - троллейбусных). В целом же в г. Белгороде и его пригородах общее количество маршрутов достигает 146. Контроль движения транспортных средств осуществляется каждым из перевозчиков в отдельности - создание единой диспетчерской службы города только планируется. Имеющиеся диспетчерские службы выполняют в основном функцию контроля нахождения транспортных средств на маршруте и архивирования принятых от них данных о местоположении и скорости движения.

Для обеспечения эффективной работы общественного транспорта на основании данных о параметрах движения подвижного состава должны и могут быть определены следующие характеристики:

- техническая скорость на участках маршрута;

- время оборота - продолжительность нахождения на маршруте;

- среднее время прибытия на остановку и его разброс;

- средняя продолжительность высадки и посадки пассажиров, его разброс;

Причём перечисленные параметры должны учитывать сезонные, суточные и других факторы.
Таким образом, сложившееся на практике расписание можно либо зафиксировать, либо внести коррективы во время отправления с начального пункта и обеспечить необходимые условия для нормального функционирования системы общественного транспорта агломерации.

\section{ПУТИ ОПРЕДЕЛЕНИЯ ПАРАМЕТРОВ ДВИЖЕНИЯ ПОДВИЖНОГО СОСТАВА ОБЩЕСТВЕННОГО ТРАНСПОРТА}

Существующие диспетчерские системы в основном обеспечивают контроль движения подвижного состава и реагируют на чрезвычайные ситуации. Другие задачи, имеющиеся у них аппаратные и программные средства в настоящее время решать, не могут.

Навигационная информация, входящая в состав сообщений, поступающих от транспортных средств в реальном масштабе времени, определяется стандартным протоколом NMEA-0183, используемым во всех типах навигаторов, трекеров и других навигационных устройств этого класса. В частности, для обработки и анализа доступны следующие данные:

- время привязки кадра данных;

- широта, долгота и высота в геодезической системе координат;

- модуль вектора скорости и курсовой угол.

Наряду с этими данными протокол NMEA0183 [5] предусматривает выдачу дополнительных данных, характеризующих качество навигационного решения, но, как правило, они в диспетчерскую систему не передаются.

Особо следует отметить качество передаваемой информации. Автомобильное навигационное оборудование предназначено для оценивая только «грубых» координат, поэтому точность координатных определений относительно невысока и согласно спецификациям производителей чипов (приёмников навигационных сигналов), интегрируемых в оборудование, составляет в горизонтальной плоскости 2,5 м (с вероятностью $50 \%$ ), что эквивалентно 7,0 м (с вероятностью $95 \%$ ). Однако эти данные характерны для работы в 


\section{А. М. Лукьянов, О. Г. Алдабаева, А. А. Лукьянов}

условиях «открытого» неба, т. е. при прямой видимости всех навигационных спутников, находящихся над горизонтом. В условиях же городов с их высотной застройкой реальные погрешности достигают в несколько раз (до десяти) больших значений. Как следствие, некоторые производители автомобильного оборудования вообще не указывают точность координатных определений [6], тем самым, не гарантируя выдаваемые данные о местоположении автомобиля.

Использование канала передачи данных GPRS приводит к тому, что поступление данных в диспетчерский центр осуществляется неравномерно. Так, навигационный приёмник выдаёт сообщения с темпом один раз в секунду, а в диспетчерском центре данные регистрируются с дискретностью от 1 секунды до 40 секунд. При этом среднее значение дискретности следования кадров с данными составляет примерно 10 секунд.

Как следствие, поступающие данные используются только для отображения в реальном времени местоположения транспортных средств на карте города (экран в диспетчерском центре).

Такое качество исходных денных не позволяет напрямую, без предварительной обработки использовать их для оценивания характеристик движения общественного транспорта. В предварительную обработку входит решение таких задач, как анализ информации и отбраковка аномальных измерений, оценивание закона движения автомобиля и интерполяция данных для восстановления пропущенных значений и ряда других.

Статистическая обработка «почищенных» И «восстановленных» данных позволяет определить техническую скорость на участках маршрута, грубую оценку времени прибытия на остановку, время оборота, соблюдение маршрута движения и ряд других характеристик. Для определения данных о времени высадки/посадки пассажиров и надёжных оценок времени прибытия на остановку необходимо гарантировано обеспечить темп поступления данных в одну секунду, что обеспечивается навигационным приёмником.
Таким образом, может быть построена модель движения общественного транспорта, составлено расписание движения в условиях реального транспортного потока и обеспечен контроль его соблюдения.

В случае использования первичной измерительной информации, выдаваемой в формате разработчика, а не NMEA-0183, и применения специализированных методов обработки информации, в том числе в дифференциальном режиме, можно существенно повысить точность и надёжность координатных определений и, тем самым, обеспечить возможность решения дополнительных задач. Это, прежде всего, оценка времени ожидания въезда на остановку при возникновении очереди, фактические места, занимаемые транспортом на остановках, в сравнении с размерами остановочного кармана, определение стиля вождения ТС и контроль соответствия ускорения/ замедления условиям комфортных пассажирских перевозок.

Для реализации всех перечисленных выше функций в настоящее время работы ведутся в двух направлениях:

- разработка программно-алгоритмического обеспечения обработки данных, накапливаемых существующими диспетчерскими системами, и ориентированное также на использование первичной измерительной информации для достижения более высокой точности результатов;

- разработка бортового оборудования на основе элементной базы, используемой для навигационной аппаратуры низкой ценовой группы, но, в отличие от существующего навигационного оборудования этой группы, позволяющая извлекать первичную измерительную информацию, в том числе, фазовую.

\section{РЕАЛИЗОВАННЫЕ ФУНКЦИИ ОПРЕДЕЛЕНИЯ ХАРАКТЕРИСТИК ДВИЖЕНИЯ ОБЩЕСТВЕННОГО ТРАНСПОРТА}

Перечисленные ранее задачи решаются в рамках системы оценивания и прогнозирования характеристик движения общественного транспорта, разрабатываемой в настоящее 
время. На данном этапе создания системы решены следующие задачи:

- определение средней скорости и среднего времени движения на рёбрах графа дорог;

- контроль соблюдения маршрута движения;

- определение фактического, реализуемого на практике, расписания движения в условиях реального транспортного потока;

- контроль соблюдения графика движения.

Исходными данными для решения задач являются граф дорожной сети, координаты вершин графа, координаты остановок, их последовательность в рамках маршрутов и данные о параметрах движения, предоставляемые навигаторами, установленными на подвижных средствах общественного транспорта.

Рассмотрим перечисленные задачи более подробно.

Средняя скорость движения общественного транспорта на ребре является одним из параметров, используемых при организации движения. Получаемые оценки могут быть используются при построении модели движения общественного транспорта, отражающей зависимость параметров его движения от времени суток, дня недели и сезона. Применение навигационных данных позволяет автоматизировать процесс сбора и обработки информации.

Оценка средней скорости движения на заданном ребре и в заданный интервал времени определяется в виде:

$$
\bar{V}_{k}\left(\tau_{i}\right)=\frac{1}{N} \sum_{n=1}^{N}\left(\frac{1}{J_{n}} * \sum_{j=1}^{J_{n}} V_{n, k}\left(t_{j}\right)\right),
$$

где $V_{n, k}\left(t_{j}\right)$ - измеренное значение скорости движения $n$-го транспортного средства на $j$-м ребре дороги;

$t_{j}$ - время привязки измеренного значения скорости движения на ребре. Время $t_{j}$ определяется по критерию:

$$
t_{j} \in\left[t_{n, k-1, i}, t_{n, k 1, i}\right]
$$

$t_{n, k-1, i}, t_{n, k, i}$ - времена последовательного проезда двух соседних вершин, ограничивающих ребро, в пределах $i$-го интервала усреднения;
$J_{n}$ - количество измеренных значений скорости за время движения в пределах ребра;

$N$ - количество транспортных средств, проехавших между вершинами за период интервала усреднения;

$\tau_{i}$ - среднее время $i$-го интервала усреднения, на которое производится оценка средней скорости.

Таким образом оценка средней скорости движения общественного транспорта формируется для каждого ребра графа дорожной сети и позволяет проанализировать и учесть при планировании пассажирских перевозок факторы «часа пик», «выходного дня» и ряд других. Зависимость средней скорости движения от различных факторов представлена в табл. 1 . На основании данных на ребрах дорожной сети можно оценивать характеристики движения не только общественного транспорта, но транспортных потоков в целом.

Навигационная информация, поступающая от трекера, позволяет автоматизировать контроль движения транспортных средств по маршруту. Наличие претензий пассажиров приводит к необходимости анализа соблюдения автобусами маршрута, выявления причин сбоев и их устранения.

В существующих диспетчерских системах движение автобуса отмечается в виде перемещающегося маркера на карте. При этом отсутствует возможность сопоставить его движение с конкретным маршрутом. Это отягощается условиями реальной обстановки, когда приходится в срочном порядке принимать оперативные решения по изменению маршрута движения. Как следствие необходим механизм, позволяющий в автоматическом режиме определять маршрут, по которому осуществлялось движение, и его соблюдение.

На рис. 1 приведена траектория движения автобуса за одни сутки. По внешнему виду траектории зачастую затруднительно однозначно определить маршруты, по которым осуществлялось движение. Реализация механизма контроля позволяет не только идентифицировать маршрут, но и указать насколько он был соблюдён (рис. 2).

Результаты, приведенные на рис. 2, требуют отдельного пояснения. Как следует из ана- 
Таблица 1

Средняя скорость движения на ребре (Стадион - Свято-Троиикий бульвар, длина: 660,1м)

\begin{tabular}{|l|c|c|c|c|}
\hline \multirow{2}{*}{\multicolumn{2}{|c|}{ Время суток }} & \multicolumn{2}{|c|}{ Ср. скорость движения, км/ч } & \multicolumn{2}{c|}{ Ср. время движения } \\
\cline { 2 - 5 } & Рабочий & Выходной & Рабочий & Выходной \\
\hline 6:30 - 9:00 (утренний пик) & 12,2 & 11,1 & $0: 03: 49$ & $0: 03: 40$ \\
\hline 9:00 - 12:00 & 13,3 & 12,4 & $0: 04: 46$ & $0: 03: 33$ \\
\hline 12:00 - 14:00 (обеденный пик) & 11,1 & 8,7 & $0: 04: 11$ & $0: 05: 07$ \\
\hline 14:00 - 16:00/16:30 & 14,9 & 8,3 & $0: 02: 48$ & $0: 04: 49$ \\
\hline 16:30 - 19:30/20:00 (вечерний пик) & 8,8 & 8,5 & $0: 04: 45$ & $0: 04: 57$ \\
\hline 20:00 - 23:00 & 18,7 & 8,6 & $0: 02: 23$ & $0: 04: 47$ \\
\hline
\end{tabular}

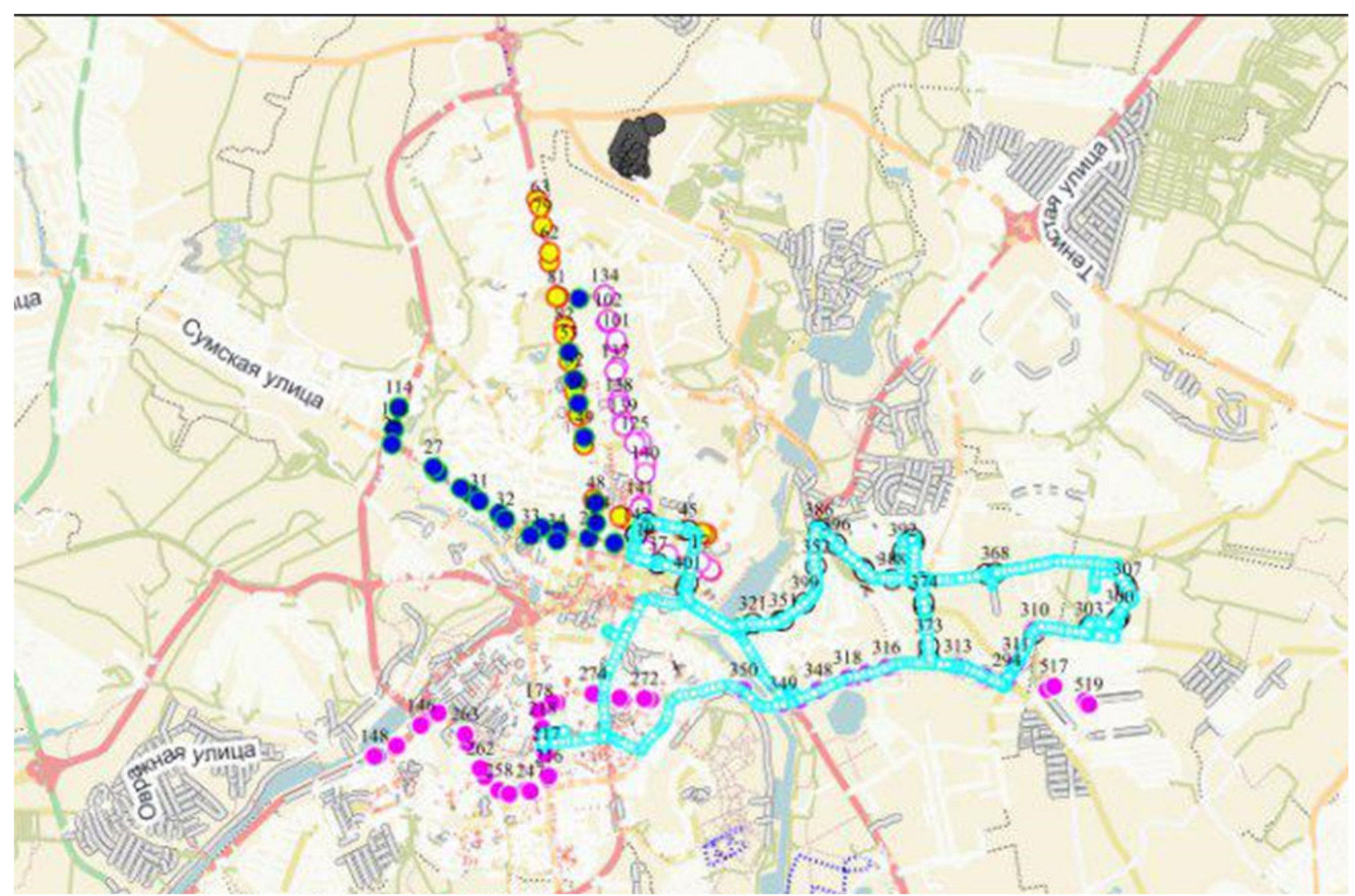

Рис. 1. Траектория движения автобуса 17 октября 2018 г

\begin{tabular}{|c|c|c|}
\hline \multicolumn{3}{|c|}{ p864кк_181017_0000-181017_2359.txt } \\
\hline \multicolumn{3}{|c|}{ CTAPT: 06:57:04 } \\
\hline Номер маршруга & Описание рейсов & Общее кол-во рейсов \\
\hline 18 & $\begin{array}{l}\text { Рейс №1. Корреляция: 0.93. Начало: 08:53:18; Окончание: 11:11:52; Длительность рейса - } 138 \text { мин; Количество пропусков - } 3 \\
\text { Рейс №2. Корреляция: 0.98. Начало: 11:13:18; Окончание: } 13: 41: 06 \text {; Длительность рейса - } 147 \text { мин; Количество пропусков - } 1 \\
\text { Рейс №3. Корреляция: 0.95. Начало: 13:42:30; Окончание: } 16: 09: 12 ; \text { Длительность рейса - } 146 \text { мин; Количество пропусков - } 2 \\
\text { Рейс №4. Корреляция: 0.86. Начало: 16:11:18; Окончание: } 18: 42: 30 ; \text { Длительность рейса - } 151 \text { мин; Количество пропусков - } 6 \\
\text { Рейс №5. Корреляция: 0.98. Начало: 18:45:06; Окончание: 20:49:50; Длительность рейса - } 124 \text { мин; Количество пропусков - } 1\end{array}$ & 5 \\
\hline Маршрут не определен & $\begin{array}{l}\text { Не уст-й рейс №1. Начало: 06:57:04; Окончание: 08:52:50;. Длительность рейса - } 115 \text { мин } \\
\text { Не уст-й рейс №2. Начало: 20:50:24; Окончание: 22:29:38;. Длительность рейса - } 99 \text { мин }\end{array}$ & 2 \\
\hline
\end{tabular}

Рис. 2. Результаты анализа движения автобуса 17 октября 2018 г

лиза траектории, автобус двигался по маршруту № 18. Есть два участка траектории, которые можно сопоставить с утренним выездом на маршрут и вечерним возвращением на место парковки. Эти участки не соответствуют ни одному из анализируемых маршрутов. В течение дня автобус сделал пять оборотов по маршруту и при этом всегда с отклонени- 


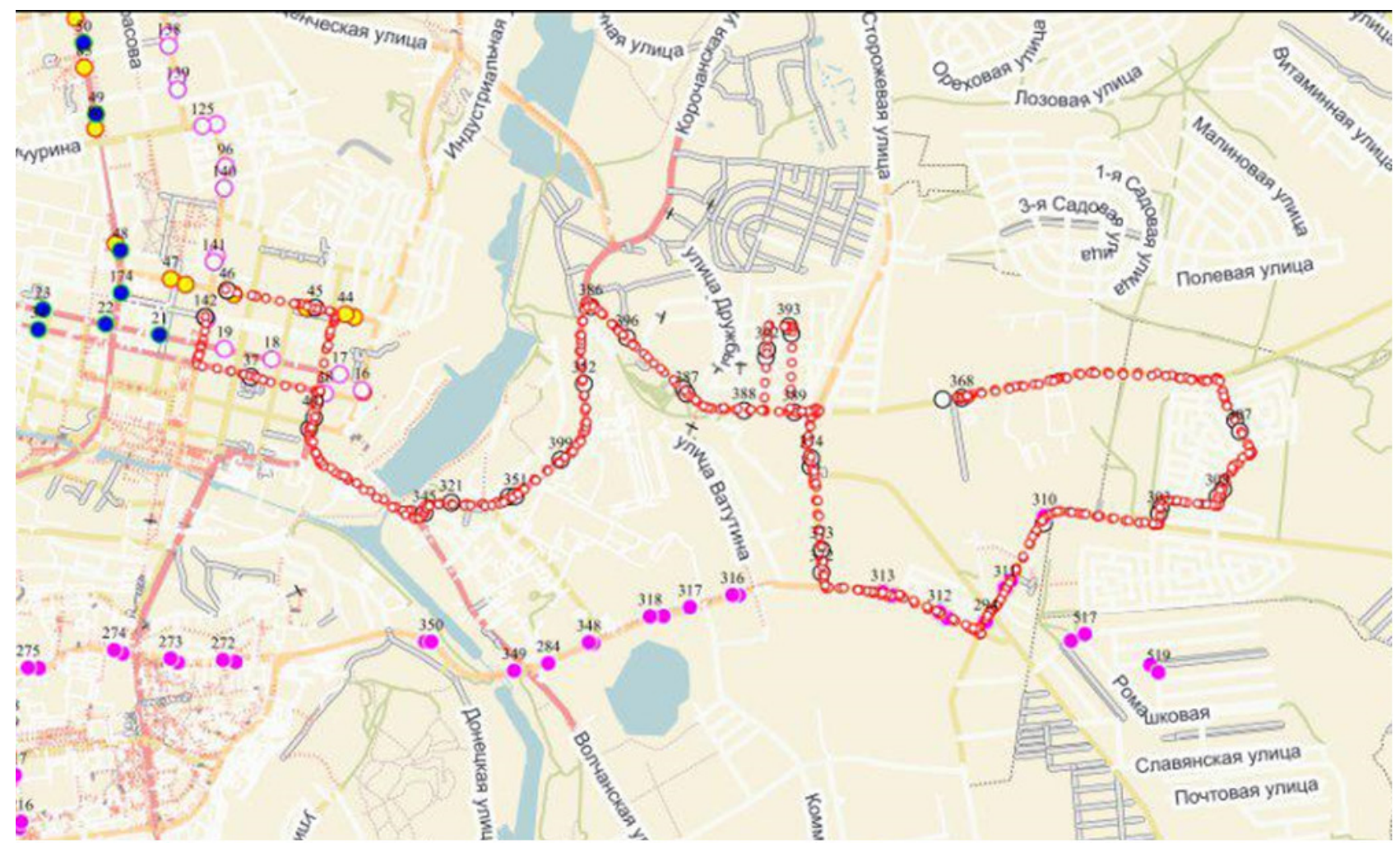

Рис. 3. Траектория движения автобуса 17 октября 2018 г. (оборот №1)

ями. Практически всегда пропускается последняя остановка на маршруте (рис. 3) - она отмечена белым в конце траектории движения. Также можно отметить пропуск ещё двух остановок, возникший после пропуска петли (рис. 4). Вариант пропуска шести остановок реализован в результате «сокращения» маршрута - при движении от конечной остановки в направлении центра города, минуя нижнюю на карте часть маршрута.

Движение маршрутного транспорта позволяет составлять реальное расписание движения и контролировать его соблюдение. Так, все транспортные средства, стартующие с начальной остановки маршрута в одно и то же время, должны прибывать на все последующие остановки также в одно и то же время. Разброс времени прибытия допустим и обусловлен дорожной обстановкой.

Алгоритм формирования расписания заключается в следующем.

Для каждой остановки маршрута формируются ежесуточные перечни времён последовательных её посещений автобусами, движущимися по данному маршруту. На следующем шаге эти перечни разделяются на две группы: рабочие дни и выходные/празднич-

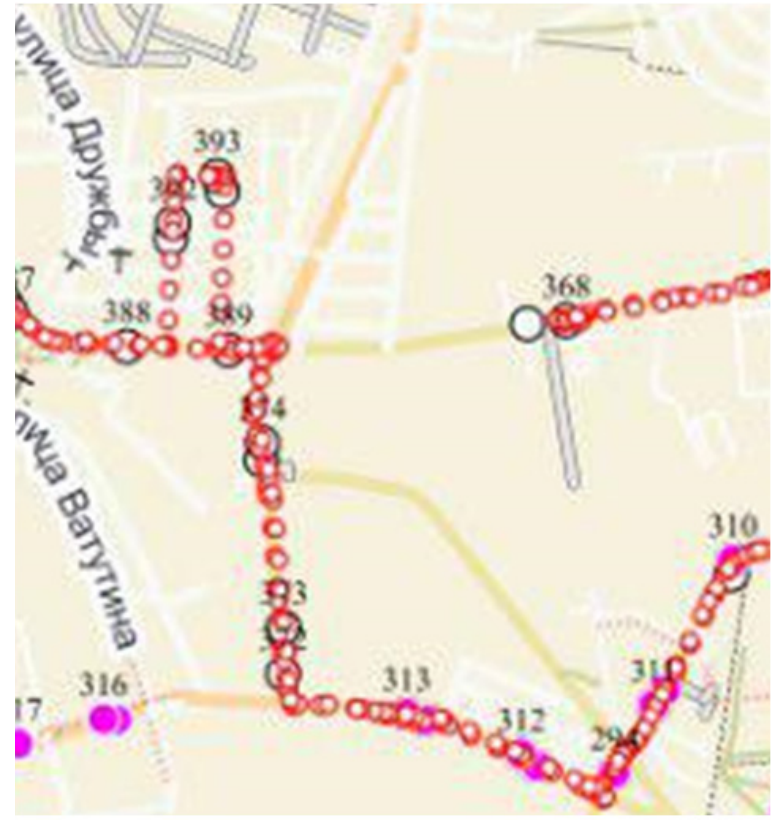

Рис. 4. Проезд напрямую, минуя искривление марирута

ные дни. По каждой из этих групп обработка данных ведётся по отдельности.

Суть обработки заключается в следующем:

- для остановки с номером $i$ осуществляется усреднение времён $j$-го посещения за $K$ дней: 


$$
T_{i, j}=\frac{1}{K} * \sum_{k=1}^{K} t_{i, j, k},
$$

где $t_{i, j, k}$ - время $j$-го прибытия на остановку с номером $i$ в $k$-й анализируемый день.

- определяется средеквадратическое отклонение времён прибытия $t_{i, j, k}$ :

$$
\sigma_{i, j}=\sqrt{\frac{1}{K-1} * \sum_{k=1}^{K}\left(t_{i, j, k}-T_{i, j}\right)^{2}}
$$

- осуществляется отбраковка аномальных данных по критерию:

$$
\left|t_{i, j, k}-T_{i, j}\right|>2 * \sigma_{i, j}
$$

- вычисляется уточнённое время прибытия на остановку:

$$
\tilde{T}_{i, j}=\frac{1}{K^{\prime}} * \sum_{\left|t_{i, j, k}-T_{i, j}\right|>2^{*} \sigma_{i, j}} t_{i, j, k} .
$$

Пример расписания, построенного на основании имеющейся информации, приведен в табл. 2.
Таблица 2

Пример расписания марирута № 7 по остановке Водстрой (№ 176)

\begin{tabular}{|c|c|}
\hline $\begin{array}{c}\text { Среднее время } \\
\text { прибытия }\end{array}$ & $\begin{array}{c}\text { СКО времён } \\
\text { прибытия, с }\end{array}$ \\
\hline $7: 34: 28$ & 0,51 \\
\hline $10: 23: 54$ & 0,49 \\
\hline $12: 06: 30$ & 0,48 \\
\hline $14: 01: 18$ & 0,46 \\
\hline $17: 26: 54$ & 0,44 \\
\hline $19: 52: 25$ & 0,43 \\
\hline
\end{tabular}

Контроль соблюдения графика движения осуществляется по результатам сравнения фактического времени прибытия на остановку с ожидаемым по расписанию. Прибытие с отклонением, превышающим допустимую величину, маркируется как отклонение от рас-

\begin{tabular}{|c|c|c|c|c|c|c|}
\hline \multicolumn{3}{|c|}{ 11.04.2017 вторник } & \multicolumn{3}{|c|}{ Заданное время для фильтра - } & ? \\
\hline \multicolumn{3}{|c|}{ Обновить решение задачи } & \multicolumn{4}{|c|}{ Времена проезда контрольных точек } \\
\hline \multicolumn{7}{|c|}{ Данные по оборотам на маршруте } \\
\hline № об-та & \multicolumn{2}{|c|}{ Время движения по маршруту } & \multicolumn{2}{|c|}{ Простой на конечной } & Интервал & \\
\hline 5 & \multicolumn{2}{|l|}{ 00:40:04 } & \multicolumn{2}{|c|}{ 00:07:45 } & 10:09:30 - 10:57:19 & | \\
\hline 6 & \multicolumn{2}{|l|}{$00: 36: 15$} & \multicolumn{2}{|c|}{$00: 10: 45$} & $10: 57: 34-11: 44: 34$ & \\
\hline 7 & \multicolumn{2}{|l|}{ 01:15:45 } & \multicolumn{2}{|c|}{ 00:12:45 } & 11:44:49 - 13:13:19 & \\
\hline \multicolumn{3}{|c|}{ Начальная точка маршрута: } & \multicolumn{2}{|c|}{ Автовокзал } & & \\
\hline \multicolumn{3}{|c|}{ Интервал движения по часам суток } & Весь день & $\checkmark$ & & \\
\hline \multicolumn{2}{|c|}{ Остановка } & \multicolumn{2}{|c|}{ Время прибытия } & По расписанию & - Средн. время & \\
\hline \multicolumn{2}{|c|}{ ул. Мичурина } & \multicolumn{2}{|c|}{$16: 37: 09$} & $16: 32: 44$ & 00:03:51 & $\wedge$ \\
\hline \multicolumn{2}{|c|}{ Энергомаш } & \multicolumn{2}{|c|}{$16: 40: 04$} & $16: 35: 21$ & $00: 02: 36$ & \\
\hline \multicolumn{2}{|l|}{ Стадион } & \multicolumn{2}{|c|}{$16: 43: 34$} & $16: 37: 48$ & $00: 02: 27$ & \\
\hline \multicolumn{2}{|c|}{ Свято-Троицкий бульвар } & \multicolumn{2}{|c|}{$16: 47: 19$} & $16: 41: 23$ & 00:03:34 & \\
\hline \multicolumn{2}{|l|}{ Водстрой } & \multicolumn{2}{|c|}{$16: 51: 34$} & $16: 44: 55$ & $00: 03: 32$ & \\
\hline \multicolumn{2}{|c|}{ ул. 5-августа } & \multicolumn{2}{|c|}{ 16:53:19 } & $16: 46: 25$ & 00:01:30 & \\
\hline ул. Мокро & усова & & $55: 58$ & $16: 49: 56$ & 00:03:31 & \\
\hline Электрокс & онтакт & & 58:04 & 16:51:33 & 00:01:36 & \\
\hline ДС Космо & & & $00: 19$ & $16: 52: 57$ & $00: 01: 23$ & $\checkmark$ \\
\hline
\end{tabular}
писания (рис. 5).

Реализованный подход к автоматизации процесса анализа навигационных данных

Рис. 5. Фактическое прибытие на остановку 
позволил решить ряд актуальных задач организации движения маршрутного транспорта. Положительные результаты оценивания были получены с использованием широкораспространённого навигационного оборудования.

Предлагаемый подход к решению задачи анализа информации автоматизировать процесс и позволяет наблюдать динамику развития ситуации с движением маршрутного транспорта, разработать рекомендации и соответствующим образом воздействовать на систему маршрутных перевозок.

Предложенный подход не только дает возможность анализа скоростных и траекторных данных большого количества транспортных средств, но и формирует необходимую информацию для понимания движения транспорта по принципу «источник» - «цель». Такой подход обеспечивает возможность динамического упреждающего управляющего воздействием на различные системы, в том числе и автоматизированные системы управления дорожным движением. При этом необходимо отметить, что предлагаемые прогнозные модели основаны на большом объеме статистической информации учитывающие различные климатические условия, динамиче-

Лукьянов Александр Михайлович - старший преподаватель кафедры программного обеспечения вычислительной техники автоматизированных систем, Белгородский государственный технологический университет им. В. Г. Шухова.

E-mail: sat_navigation@mail.ru

Алдабаева Ольга Геннадиевна - магистрант кафедры программного обеспечения вычислительной техники автоматизированных систем, Белгородский государственный технологический университет им. В. Г. Шухова.

E-mail: olga-aog@rambler.ru

Лукьянов Алексей Александрович - магистрант кафедры программного обеспечения вычислительной техники автоматизированных систем, Белгородский государственный технологический университет им. В. Г. Шухова. E-mail: sat_navigation@mail.ru ские характеристики транспортных средств, реальные геометрические показатели улично-дорожной сети.

\section{СПИСОК ЛИТЕРАТУРЫ}

1. Яндекс карты. Пробки [Электронный pecypc] - Режим доступа: https://yandex.ru/ maps/probki (Дата обращения: 02.03.2018).

2. Городской транспорт Великого Новгорода [Электронный ресурс] - Режим доступа: http://transport.nov.ru/urban_trans/ (Дата обращения: 02.03.2018).

3. Мониторинг общественного транспорта города Кирова [Электронный ресурс] - Peжим доступа: https://m.cdsvyatka.com/index_ rasp.php (Дата обращения: 02.03.2018).

4. СПб ГКУ «Организатор Перевозок» города Санкт-Петербурга [Электронный ресурс] - Режим доступа: http://orgp.spb.ru/bus/ (Дата обращения: 02.03.2018).

5. https://www.irz.ru/uploads/files/226_1.pdf (Дата обращения: 02.03.2018).

6. Мониторинг общественного транспорта города Кирова [Электронный ресурс] Режим доступа: http://www.auto.shtrih-m.ru/ produktyi-i-uslugi/taxografyi/sravnenie.html (Дата обращения: 02.03.2018).

Lukyanov Alexander Mikhailovich - Senior lecturer, Belgorod State Technological University named after V.G. Shukhov.

E-mail: sat_navigation@mail.ru

Aldabayeva Olga Gennadievna - Master student, Belgorod State Technological University named after V.G. Shukhov.

E-mail: olga-aog@rambler.ru

Lukyanov Alexey Alexandrovich - Master student, Belgorod State Technological University named after V.G. Shukhov.

E-mail: sat_navigation@mail.ru 